\title{
Protective Effect of Ilex spinigera and Gleditsia caspica Extracts against Drug-Induced Hemolysis in Glucose-6-phosphate dehydrogenase-deficient Patients
}

\author{
Maryam Mohadjerani (PhD) \\ Department of Molecular and Cell \\ Biology, Faculty of Basic Sciences, \\ University of Mazandaran, Babolsar, \\ Iran \\ Mansoore Damanjany (MSc) \\ Department of Molecular and Cell \\ Biology, Faculty of Basic Sciences, \\ University of Mazandaran, Babolsar, \\ Iran \\ Corresponding author: Maryam \\ Mohadjerani \\ Email: m.mohajerani@umz.ac.ir \\ Tel: +98-1135302455 \\ Address: Faculty of Basic Sciences, \\ University of Mazandaran, Babolsar, \\ Iran \\ Received: 05 May 2018 \\ Revised: 05 Sep 2018 \\ Accepted: 25 Sep 2018

\section{(c) (1) (3)}

This work is licensed under a Creative Commons Attribution 4.0 License.

\section{ABSTRACT}

Background and Objectives: Glucose-6-phosphate dehydrogenase (G6PD) deficiency is the most common X-linked disorder of human erythrocytes in which cells are susceptible towards hemolytic changes and could be destroyed by peroxides. Extracts of Ilex spinigera and Gleditsia caspica leaves have excellent free radical scavenging activity. We investigated the protective effects of these extracts against hemolysis induced by some drugs in G6PI-deficient erythrocytes.

Methods: Blood samples were collected from males with and without 66PD deficiency. Hemolysis induced by aspirin, phenylhydrazine hydrochloride and phenacetin was assessed in the presence and absence of the extracts. The amount of released hemoglobin was determined by reading absorbance at $540 \mathrm{~nm}$.

Results: The methanol extract of 6 . caspica had significant protective effects against phenacetin-induced hemolysis in G6PI)-deficient human erythrocytes $(\mathrm{P}<0.05)$. However, the I. spinigera extract had no significant anti-hemolytic effects on these cells.

Conclusion: 0ur findings suggest that the extract of G. caspica could be a potential drug with antioxidant and anti-hemolytic properties for patients with G6PD deficiency.

Keywords: Antihemolytic activity, Medicinal plant, G6PD deficiency, Gleditsia caspica, Ilex spinigera. 


\section{INTRODUCTION}

Glucose-6-phosphate dehydrogenase (G6PD, EC: 1.1.1.49) is an important and highly conserved enzyme in the pentose phosphate metabolic pathway (1). It catalyzes the simultaneous oxidation of glucose-6phosphate to 6-phosphogluconate and reduction of $\mathrm{NADP}^{+}$to NADPH. This process leads to the conversion of oxidized glutathione to reduced glutathione (GSH), which protects human cells from oxidative damage (2).

A free radical is any molecular species capable of independent existence that contains one or more unpaired electrons. The oxygen radicals and related species, singlet oxygen, superoxide radicals and hydrogen peroxide are known as reactive oxygen species (ROS). Imbalance between ROS and antioxidants causes oxidative stress, which can lead to cell damage (3). Oxidative stress could be due to increased ROS production, and toxicity caused by drugs (e.g. trimethoprim, sulfamethoxazole, dapsone, primaquine, etc.), acute infection (acute hepatitis A, hepatitis B, cytomegalovirus, pneumonia, etc.), or some foods (fava beans) can also cause G6PD deficiency-related hemolysis (4).

Primaquine is the only drug currently licensed for the radical treatment of Plasmodium vivax malaria. However, it can cause severe hemolysis in individuals with G6PD deficiency, a common genetic disorder that is positively associated with $P$. vivax incidence. G6PD deficiency is largely asymptomatic until individuals are exposed to external sources of oxidative stress, including certain nutrients and drugs, but most notably fava beans (5). GSH is a major antioxidant that protects cells against oxidative damage (6).

A proposed mechanism for the lack of resistance of G6PD-deficient RBCs to oxidative stress is shown in figure 1 . The interaction of drugs with RBCs leads to hydrogen peroxide production, either directly or through ROS. GSH can remove the produced hydrogen peroxide in the presence of glutathione peroxidase. The produced oxidized glutathione will be reduced to regenerate GSH through the activity of glutathione reductase. Then, continuous reduction of NADP to NADPH relies on the G6PD activity (7).

Antioxidants, flavonoids and phenolic compounds act as a major defense system against radical-mediated toxicity and free radical-induced damage (8). According to Flora Iranica, Ilex spinigera and Gleditsia caspica are plants native to the forests of northern Iran, particularly in Guilan, Golestan, Mazandaran and Azerbaijan Provinces (9). These two plants have numerous antioxidant, biological and therapeutic properties $(10,11)$. The present study investigates effects of $I$. spinigera and $G$. caspica extracts on hemolysis induced by aspirin, phenacetin and phenylhydrazine hydrochloride in G6PDdeficient human erythrocytes.

\section{MATERIALS AND METHODS}

Leaves of I. spinigera and G. caspica were collected from Mazandaran forests. The specimens of I. spinigera (voucher Nr.4034) and G. caspica (voucher Nr. 5502) were obtained from the Herbarium of University of Mazandaran. The powdered plants samples (20 grams) were extracted using a method described in our previous work (10).

Total phenolic and flavonoid content of each extract were assessed using the FolinCiocalteu phenol and aluminum chloride colorimetric methods $(12,13)$. All chemicals used in the study were of analytical grade and purchased from Fluka, Sigma and Merck Co. All kits were obtained from Kimia Pajouhan Co., Iran. We randomly collected 315 blood samples from men in Babolsar. G6PD deficiency was screened in the samples using the fluorescent spot test (Kimia Pajouhan kits) (14). To determine the concentration of the drugs that could produce approximately $50 \%$ hemolysis in the G6PD-deficient erythrocytes, suspension aliquots were incubated with varying amounts of the drugs (0 to $4 \mathrm{mM}$ ) (15). The percentage of hemolysis was calculated by dividing the absorbance reading (at $540 \mathrm{~nm}$ ) from the experimental tubes by the mean value of complete hemolysis induced by distilled water multiplied by 100 . Data were expressed as means \pm standard deviation. All measurements were replicated three times. Half-maximal inhibitory concentration $\left(\mathrm{IC}_{50}\right)$ values were calculated via linear regression analysis. One-way analysis of variance (ANOVA) and Tukey's post-test were carried out for comparison of data. P-values less than 0.05 were considered statistically significant. 
Figure 1. A plausible series of reactions that could explain the lack of resistance of G6PD-deficient red blood cells (RBCs) to oxidative stress (7)

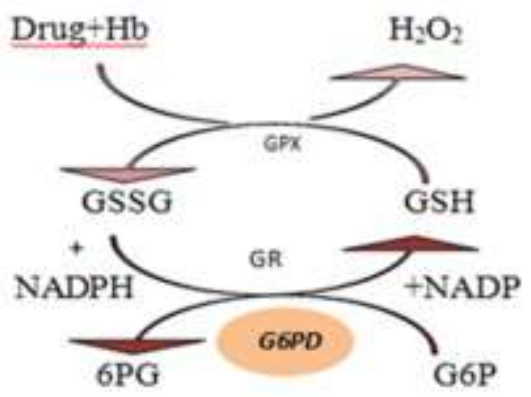

\section{RESULTS}

The extraction yield and total phenolic and flavonoid content of the extracts are reported in table 1.

The total phenolic content was higher in the methanol extract of $G$. caspica $(0.123 \pm 0.003$ $\mathrm{mg}$ gallic acid equivalents per gram of dried leaves). Similarly, the total flavonoid content was higher in the G. caspica extract
$(0.459 \pm 0.007 \mathrm{mg}$ quercetin equivalents per gram of dried leaves).

Our results revealed that the frequency of G6PD deficiency is $15 \%$ among males in Babolsar, Iran. Figures 2-4 show that G6PDdeficient and normal human erythrocytes undergo hemolysis when exposed to aspirin, phenylhydrazine hydrochloride and phenacetin.

Table 1- Total phenolic and flavonoid content of $G$. caspica and $I$. spinigera extracts

\begin{tabular}{cccc}
\hline Extract & Total phenolic content* & Total flavonoid content** & Extraction yield (w/w \%) \\
\hline G. caspica & $\mathbf{0 . 1 2 3} \pm \mathbf{0 . 0 0 3}$ & $\mathbf{0 . 4 5 9} \pm \mathbf{0 . 0 0 7}$ & 10.35 \\
I. spinigera & $\mathbf{0 . 0 4 2} \pm \mathbf{0 . 0 0 4}$ & $\mathbf{0 . 0 3 0} \pm \mathbf{0 . 0 0 5}$ & 19.54 \\
\hline
\end{tabular}

* mg gallic acid equivalents per gram of dried leaves

** mg mg quercetin equivalents per gram of dried leaves

Figure 2- Hemolysis of G6PD-deficient and -sufficient erythrocytes after 2 hours of incubation at $37^{\circ} \mathrm{C}$ with different concentrations of aspirin. Water-induced hemolysis was considered $100 \%$.

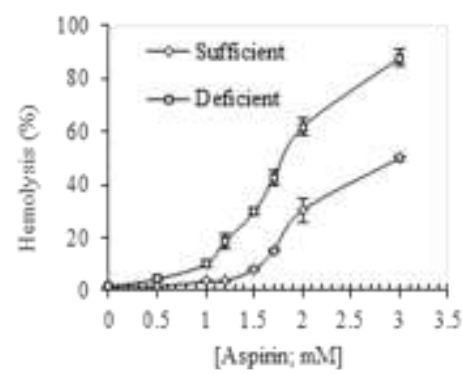

Figure 3- Hemolysis of G6PD-deficient and -sufficient erythrocytes after 2 hours of incubation at $37^{\circ} \mathrm{C}$ with different concentrations of phenylhydrazine hydrochloride. Water-induced hemolysis was considered as $100 \%$.

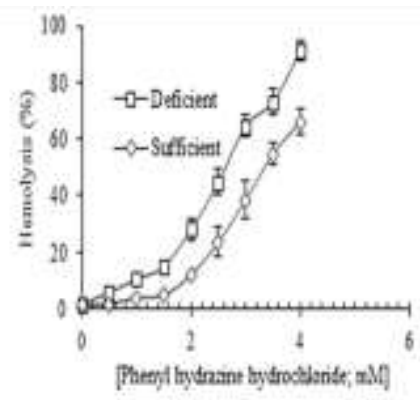


The degree of hemolysis is dependent on concentration of the hemolytic agent present in the medium. The I. spinigera extract had no significant effect on the hemolysis induced by aspirin, phenacetin and phenylhydrazine hydrochloride in G6PD-deficient erythrocytes $(\mathrm{P}>0.05)$. However, the plant extract showed higher anti-hemolytic activity at higher concentrations (Figure 5). The I. spinigera extract at a high dose $(600 \mu \mathrm{g} / \mathrm{ml})$ did not have any side effects on the RBCs when used alone. The inhibitory activity of the extract was compared with that of the positive control, glutathione $(0.5 \mathrm{mM})$.

In all cases except for phenylhydrazine hydrochloride, glutathione had significant inhibitory activity against the tested drugs $(\mathrm{P}<0.05)$.

Figure 4- Hemolysis of G6PD-deficient and -sufficient erythrocytes after 2 hours of incubation at $37^{\circ} \mathrm{C}$ with different concentrations of phenacetin. Water-induced hemolysis was considered as $100 \%$.

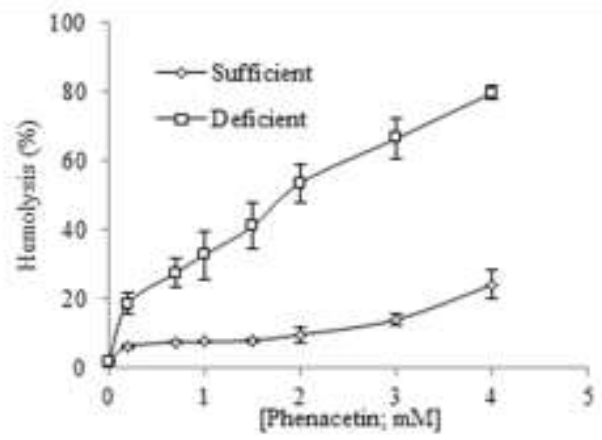

Table 2- Concentrations of aspirin, phenylhydrazine hydrochloride and phenacetin that could produce approximately $50 \%$ hemolysis.

\begin{tabular}{ccc}
\hline Hemolysis (50\%) & G6PD-sufficient erythrocytes & G6PD-deficient erythrocytes \\
\hline Aspirin & $2.9 \mathrm{mM}$ & $1.96 \mathrm{mM}$ \\
Phenyl hydrazine hydrochloride & $3.2 \mathrm{mM}$ & $2.55 \mathrm{mM}$ \\
Phenacetin & $>4 \mathrm{mM}$ & $2.09 \mathrm{mM}$
\end{tabular}

Figure 5- Effects of different concentrations of the I. spinigera extract on hemolysis induced by aspirin (A), phenacetin (B) and phenylhydrazine hydrochloride (C) in G6PD-deficient erythrocytes (Control: sample not treated with extract or drug, glutathione: positive control, and *: sample without drug treatment).

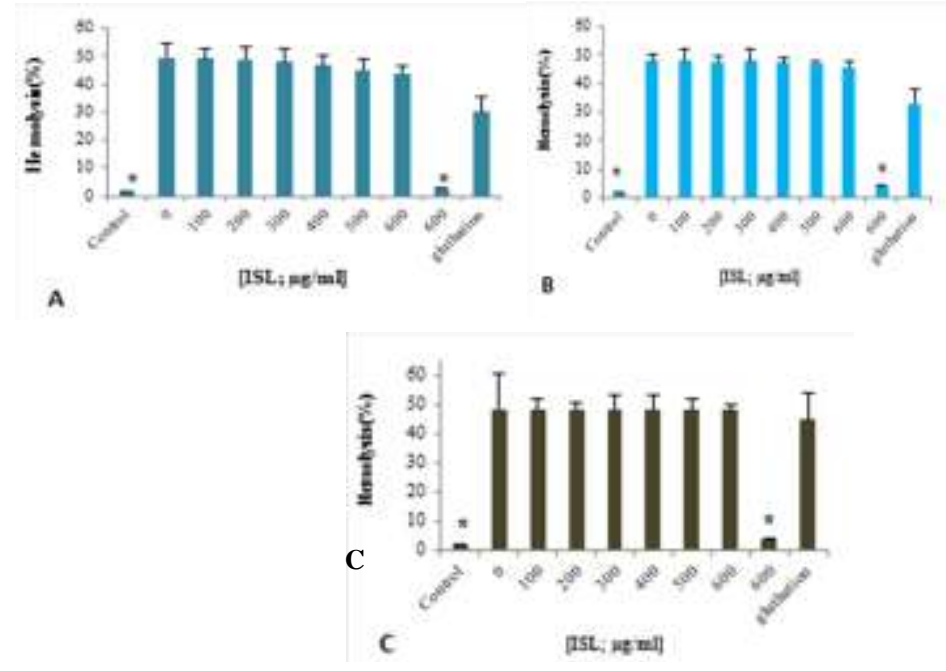


The G. caspica extract showed antihemolytic activity in a dose-dependent manner (Figure 6). This activity was significant only in the case of phenacetin-induced hemolysis $(\mathrm{P}<0.05)$.
High concentrations of this extract had no side effect on the RBCs when used alone. The inhibitory activity of glutathione was significant against all tested drugs except for phenylhydrazine hydrochloride.

Figure 6- Effects of different concentrations of the $G$. caspica extract on hemolysis induced by aspirin (A), phenacetin (B) and phenylhydrazine hydrochloride (C) in G6PD-deficient erythrocytes (Control: sample not treated with extract or drug, glutathione: positive control, and *: sample without drug treatment).

\section{DISCUSSION}

It is well established that the biological and therapeutic activities of a plant relies primarily on its contents (16). In a previous study, we demonstrated that the G. caspica extract with a higher phenolic and flavonoid content than I. spinigera, could effectively protect G6PD-deficient erythrocytes against hemolysis induced by 2,2'-azobis(2amidinopropane) dihydrochloride. In the present study, the G. caspica extract showed similar in vitro protective effects against phenacetin-induced hemolysis. While mechanisms of hemolysis induced by these substances are not clear, a common quality shared by most hemolytic agents is the ability to produce oxidative cell damage (17).

RBC loses its nucleus, ribosomes, mitochondria and other organelles during its development process. As a result, the mature circulating erythrocytes cannot replicate or synthesize protein, and have limited number of enzymatic reactions. G6PD-deficient erythrocytes do not have the adequate capacity to destroy peroxides hence cannot withstand hemolysis. Under oxidative stress, GSH depletion becomes quicker than GSH regeneration (18).

Most drugs that can induce hemolysis in G6PD-deficient erythrocytes possess oxidative properties. Although aspirin is classified as a mild hemolytic agent, it might cause severe hemolysis of erythrocytes even at small therapeutic doses. The hemolytic effects of aspirin have been shown to be even greater in G6PD-deficient individuals (19). Shahidi proved that a metabolic intermediate of acetylsalicylic acid inhibits G6PD in subjects with severe G6PD-deficiency (20) Phenylhydrazine increases generation of hydrogen peroxide when incubated with RBCs (19). Incubation of normal and G6PD-deficient RBCs with phenylhydrazine induces a decrease in adenosine triphosphate level and a subsequent increase in adenosine diphosphate and adenosine monophosphate levels (21). Phenylhydrazine reacts with oxyhemoglobin and generates hydrogen peroxide or equivalent free radicals, which in turn oxidize hemoglobin and other proteins (22). Hemolytic anemia induced by phenacetin may be acute or chronic. The main features of phenacetininduced chronic hemolytic anemia are alteration of RBC morphology and permeability as well as formation of methemoglobin, sulfhemoglobin and Heinz bodies (23). Since the G. caspica leaf extract has strong radical scavenging activity, it could act as a defense mechanism against phenacetin-induced hemolysis in G6PDdeficient erythrocytes. Erythrocytes have a high content of polyunsaturated lipids, which along with the rich oxygen supply and transition metals make them more susceptible to lipid peroxidation (24). Any process that increases peroxidation in membrane lipids also increases erythrocyte fragility (25). Although 
various free radical species are formed via the interaction of xenobiotics with erythrocytes, several membrane systems can provide protection against free radicals damage. Among them, superoxide dismutase, glutathione peroxidase and catalase are the main endogenous enzymatic defense systems (26).

Although it is not clear whether the G. caspica can boost these membrane protective mechanisms, but it could slow down the depletion of these components, thus enhancing their membrane preserving effect in G6PDdeficient erythrocytes. Moreover, some studies suggested that flavonoids might have the ability to delay consumption of some endogenous antioxidants in the human body (27). Free radicals-induced structural changes in G6PD-deficient erythrocyte membrane are not entirely understood (28).

It is known that phenacetin has the potential to affect cell membrane permeability and cause oxidative cell damage (29). Therefore,

\section{REFERENCES}

1. Aletayeb SM, Chomeili B, Taheri M, Kajbaf TZ, Hakimzadeh M, Aminzadeh M, Moghadam MS, Maleki S. The high incidence of acute hemolysis due to favism in Ahvaz, Iran-clinical features and laboratory findings. Asian Pacific Journal of Tropical Medicine. 2010; 3(5): 399-401.

2. Yang Y, Li Z, Nan P, Zhang X. Drug-induced glucose-6-phosphate dehydrogenase deficiency-related hemolysis risk assessment. Computational biology and chemistry. 2011; 35(3): 189-92.

3. Halliwell B. Reactive species and antioxidants. Redox biology is a fundamental theme of aerobic life. Plant Physiology. 2006; 141(2): 312-22.

4. Dehghanifard A, Mortazavi Y, Saki N, FarshdustiHagh M. Molecular aspects of glucose-6-phosphate dehydrogenase deficiency in Iran. Zahedan Journal of Research in Medical Sciences. 2012; 14(7): 1-7.

5. Lee J, Im Kim T, Kang JM, Jun H, Lê H G, Thái TL, $\mathrm{Na}$ B K. Prevalence of glucose-6-phosphate dehydrogenase (G6PD) deficiency among malaria patients in Upper Myanmar. BMC infectious diseases. 2018; 18(1):131. doi: 10.1186/s12879-0183031-y.

6. Georgakouli K, Deli CK, Zalavras A, Fatouros IG, Kouretas D, Koutedakis Y, et al. $\alpha$-Lipoic acid supplementation up-regulates antioxidant capacity in adults with G6PD deficiency. Food and chemical toxicology. 2013; 61: 69-73.

7. Beutler E. Glucose-6-phosphate dehydrogenase deficiency: a historical perspective. Blood. 2008; 111(1): 16-24. the anti- hemolytic effect of the G. caspica extract might be due to its membranestabilizing effects as well as its free radical scavenging activity.

\section{CONCLUSION}

We demonstrated that the methanol extract of Gleditsia caspica has strong antioxidant and anti-hemolytic properties, and therefore can be used as a potential drug for subjects with G6PD deficiency. In addition, the extract has no side effects on erythrocytes. However, more studies are required to confirm these results.

\section{ACKNOWLEDGMENTS}

The authors would like thank the Research Council of Mazandaran University for financial support.

\section{CONFLICT OF INTEREST}

The authors declare that there is no conflict of interest.

8. Kumar S, Kumar D, SAROHA K, Singh N, Vashishta B. Antioxidant and free radical scavenging potential of Citrullus colocynthis (L.) Schrad. methanolic fruit extract. Acta Pharmaceutica. 2008; 58(2): 215-20. doi: 10.2478/v10007-008-0008-1.

9. Rechinger KH. Aquifoliaceae in KH Rechinger. Flora Iranica. Vol 25, Akademische Druck. Verlagsanstalt. Graz. 1977.

10. Mohadjerani M, Damanjany M. Inhibition of Hemolysis of Red Blood Cells by Citrullus colocynthis, Ilex spinigera and Gleditsia caspica Extracts. Journal of Medicinal Plants. 2015; 14(54): 134-45.

11. Mohadjerani M, Vosoghi Roodgar M. In-vitro evaluation of protective effects on DNA damage and antioxidative activities of Ilex Spinigera Loes. extracts. Iranian Journal of Pharmaceutical Research. 2016; 15(1): 283-292.

12. Singleton VL, Rossi JA. Colorimetry of total phenolics with phosphomolybdic-phosphotungstic acid reagents. American journal of Enology and Viticulture. 1965; 16(3): 144-58

13. Marinova D, Ribarova F, Atanassova M. Total phenolics and total flavonoids in Bulgarian fruits and vegetables. Journal of the university of chemical technology and metallurgy. 2005; 40(3): 255-60.

14. Beutler E, Mitchell M. New rapid method for the estimation of red cell galactose-1-phosphate uridyl transferase activity. The Journal of laboratory and clinical medicine. 1968; 72(3): 527-32. 
15. Sharma SC, Sharma S, Gulati OP. Pycnogenol prevents haemolytic injury in G6PD deficient human erythrocytes. Phytotherapy Research. 2003; 17(6): 671674.

16. Umar MI, Asmawi MZ, Sadikun A, Abdul Majid AMS, Atangwho IJ, Khadeer Ahamed MB , et al. Multiconstituent synergism is responsible for antiinflammatory effect of Azadirachta indica leaf extract. Pharmaceutical Biology. 2014; 52(11): 1411-1422.

17. Beutler E, Lisker R, Kuhl W. Molecular biology of $G$ 6 PD variants. Biomedica biochimica acta. 1990; 49(23): S236-41.

18. Butnariu M, Samfira I. Free radicals and oxidative stress. J Bioequiv Availab. 2012; 4: 0975-851.

19. De Leeuw NK, Shapiro L, Lowenstein L. Druginduced hemolytic anemia. Annals of internal medicine. 1963 Apr 1;58(4):592-607.

20. Shahidi NT, Westring DW. Acetylsalicylic acidinduced hemolysis and its mechanism. The Journal of clinical investigation. 1970; 49(7): 1334-40.

21. Welt SI, Jackson EH, Kirkman HN, Parker JC. The effects of certain drugs on the hexose monophosphate shunt of human red cells. Ann N Y Acad Sci. 1971; 179(1): 625-35.

22. Mohler DN, Williams WJ. The effect of phenylhydrazine on the adenosine triphosphate content of normal and glucose-6-phosphate dehydrogenasedeficient human blood. J Clin Invest. 1961; 40(9): 17351742.
23. Millar J, Péloquin R, de Leeuw NK. Phenacetininduced hemolytic anemia. Can Med Assoc J. 1972; 106(7): 770-775.

24. Chiu D. Peroxidative reactions in red cell biology. Free radicals in biology. 1982; 5: 115-60.

25. Brzezińska-Ślebodzińska E. Erythrocyte osmotic fragility test as the measure of defence against free radicals in rabbits of different age. Acta Veterinaria Hungarica. 2001; 49(4): 413-9.

26. Miki M, Tamai H, Mino M, Yamamoto Y, Niki E. Free-radical chain oxidation of rat red blood cells by molecular oxygen and its inhibition by $\alpha$-tocopherol. Arch Biochem Biophys. 1987; 258(2): 373-80.

27. Lotito SB, Fraga CG. (+)-Catechin prevents human plasma oxidation. Free Radic Biol Med. 1998; 24(3): 435-41.

28. Hoffman JF. On red blood cells, hemolysis and resealed ghosts. Adv Exp Med Biol. 1992; 326: 1-15.

29. Knopik-Skrocka AG, Bielawski J. The mechanism of the hemolytic activity of polyene antibiotics. Cell Mol Biol Lett. 2002; 7(1): 31-48. 